\title{
Growth and characterization of CdTe:Ge:Yb
}

\author{
N.V. Sochinskii ${ }^{a, *}$, E. Saucedo ${ }^{\mathrm{b}}$, M. Abellan ${ }^{\mathrm{a}}$, J. Rodríguez-Fernández $^{\mathrm{a}}$, P. Hidalgo $^{\mathrm{c}}$, \\ J. Piqueras ${ }^{\mathrm{c}}$, C.M. Ruiz ${ }^{\mathrm{b}}$, V. Bermúdez ${ }^{\mathrm{b}}$, E. Diéguez ${ }^{\mathrm{b}}$ \\ ${ }^{a}$ Instituto de Microelectrónica de Madrid, CNM-CSIC, Parque Tecnológico de Madrid, Tres Cantos, 28760 Madrid, Spain \\ ${ }^{\mathrm{b}}$ Departamento de Física de Materiales, Universidad Autónoma de Madrid, Ctra. Colmenar km. 14, Madrid 28049, Spain \\ ${ }^{\mathrm{c}}$ Departamento de Física de Materiales, Facultad de Fisicas, Universidad Complutense, E-28040 Madrid, Spain
}

Available online 21 November 2007

\begin{abstract}
Cadmium telluride (CdTe) crystals and epitaxial layers were grown by the vertical Bridgman method and vapor-phase epitaxy, respectively, to obtain the high-resistive material suitable for X-and gamma-ray detectors. The crystals and layers were doped with Ge at the concentration of $5 \times 10^{17} \mathrm{~cm}^{-3}$ and co-doped with the rare element $\mathrm{Yb}$ at the concentration range from $1 \times 10^{17}$ to $1 \times 10^{19} \mathrm{~cm}^{-3}$. The $\mathrm{CdTe}: \mathrm{Ge}: \mathrm{Yb}$ samples were studied by the structural and electrical characterization techniques, low-temperature photoluminescence (PL) and cathodoluminescence (CL) spectroscopy and CL imaging.

Experimental findings testify that homogeneous crystals and layers of reasonably good structural quality can be grown with the $\mathrm{Yb}$ concentration below the value of $5 \times 10^{18} \mathrm{~cm}^{-3}$ that is estimated to be the limit for Yb solubility in CdTe:Ge: $\mathrm{Yb}$. These findings seem to be related with the purification effect caused by the interaction of the Yb dopant with the group I residual impurities.
\end{abstract}

(C) 2007 Elsevier B.V. All rights reserved.

PACS: 81.10.f; 81.05.d

Keywords: A2. Bridgman technique; A3. Vapor-phase epitaxy; B1. CdTe; B2. Semiconducting II-VI

\section{Introduction}

Doped cadmium telluride (CdTe) is a promising semiconductor material for important applications such as room-temperature $\mathrm{X}$ - and gamma-radiation detectors, photonics, radiation sensors, infrared imaging, etc. [1]. Although there has been considerable progress in the growth and characterization of doped CdTe bulk crystals and epitaxial layers by different techniques, their commercial use is still limited by the numerous technological problems (for example, see Refs. [2-6]).

In the particular case of CdTe for room-temperature radiation detectors, it has been demonstrated that $\mathrm{CdTe}$ crystals doped with $\mathrm{Ge}$, that were grown by the Bridgman method, are semi-insulating and of reasonably good structural quality [7]. More recently, we have shown that the transport charge properties of Ge-doped CdTe could be

\footnotetext{
${ }^{*}$ Corresponding author. Tel.: + 34918060700 ; fax: +34918060701 .

E-mail address: ms@imm.cnm.csic.es (N.V. Sochinskii).
}

improved by the co-doping with rare earth elements, e.g. ytterbium [8].

In our previous studies, we have demonstrated that the combined use of photoluminescence (PL) and cathodoluminescence (CL) spectroscopy and imaging techniques are powerful tools to investigate Ge-doped $\mathrm{CdTe}$ crystals $[9,10]$. In the present paper, we report the results of luminescence studies of the energetic location and spatial distribution of structural defects in CdTe crystals co-doped with $\mathrm{Ge}$ and $\mathrm{Yb}$, and compare these results with the structural and electrical characterization data.

\section{Experimental procedure}

CdTe crystals co-doped with Ge and $\mathrm{Yb}$ were grown by the vertical Bridgman method in sealed high-purity quartz ampoules using electronic-grade raw materials. Prior to the growth process, the CdTe:Ge: $\mathrm{Yb}$ mixture was synthesized and homogenized at $1200{ }^{\circ} \mathrm{C}$ in an oscillating furnace for $20 \mathrm{~h}$. During the Bridgman growth, the CdTe:Ge:Yb 
crystals were pulled down at the rate of $0.5-2 \mathrm{~mm} / \mathrm{h}$ under a thermal gradient of $5-7^{\circ} \mathrm{C} / \mathrm{cm}$. Based on the results of our previous studies on the growth and properties of doped $\mathrm{CdTe}[4,6,8,11]$, the nominal Ge concentration was maintained constant at the value $5 \times 10^{17} \mathrm{~cm}^{-3}$ in all CdTe:Ge: $\mathrm{Yb}$ crystals and the $\mathrm{Yb}$ concentration was varied in the range from $10^{17}$ to $10^{19} \mathrm{~cm}^{-3}$. The crystals were sliced perpendicularly to the growth direction into the characterization samples of $10 \times 10 \mathrm{~mm}^{2}$ area and $1 \mathrm{~mm}$ thickness, which were chemically polished in the $5 \%$ bromine-methanol solution and washed to remove the surface damage.

Using these CdTe:Ge: $\mathrm{Yb}$ crystals as evaporation sources, the vapour-phase epitaxy (VPE) growth of CdTe:Ge:Yb 1-10- $\mu$ m-thick layers was carried out on the $\mathrm{Si}(100)$ substrates at temperatures in the range of $500-750{ }^{\circ} \mathrm{C}$ and the growth rate in the range of $1-10 \mathrm{~nm} / \mathrm{s}$. The VPE experiments were made in the modified commercial Pfeiffer Classic 500 vacuum coating system.

The CdTe:Ge:Yb crystals and layers were studied by a Philips XL30 scanning electron microscope (SEM) equipped with an energy-dispersion $\mathrm{X}$-ray analyzer (EDAX) and a Seifert XRD-3000TT two axis X-ray diffractometer (XRD) for structural inspection and by the $I-V$ and quadrature frequency-resolved photoconductivity (QFRP) measurements for electrical characterization. The dopant and residual impurity concentrations were measured using inductively coupled plasma mass spectroscopy (ICP-MS) with a mass spectrometer ELAN-6000 (PE-Sciex).

The PL characterization was performed at $10 \mathrm{~K}$ using a Spectra Physics 2020-05 Ar laser as excitation source, with a $16810.22 \mathrm{~m}$ Spex spectrometer coupled to a North Coast Scientific Corporation Ge detector EO-817L.

The CL measurements were performed with a cooled ADC germanium detector, in a Hitachi S-2500 SEM at liquid nitrogen temperature and at an electron accelerating voltage of $20 \mathrm{kV}$.

\section{Results and discussions}

Based on the SEM's data, the lowest limit for Yb doping in $\mathrm{CdTe}: \mathrm{Ge}: \mathrm{Yb}$ crystals was estimated to be in the range of $1 \times 10^{16}-1 \times 10^{17} \mathrm{~cm}^{-3}$. It is comparable with the concentration of the group I residual impurities like $\mathrm{Na}, \mathrm{Li}, \mathrm{Cu}$ and $\mathrm{K}$, which are the most electrically active contaminants in CdTe. The upper doping limit for $\mathrm{Yb}$ was determined by the combined SEM, EDAX and XRD structural inspection. It was found that the CdTe:Ge:Yb homogeneous crystals and layers of reasonably good structural quality could be grown with the $\mathrm{Yb}$ concentration below the value of about $5 \times 10^{18} \mathrm{~cm}^{-3}$, depending on the growth process details.

After electrochemical gold contacts deposition, the $I-V$ characterization was carried out and the photoconductive response of CdTe:Ge:Yb samples was measured by the QFRP technique as a function of excitation light frequency
[12]. As usual, it was seen that the photoconductivity increases from low frequencies up to the maximum photocurrent value $f_{\max }$ and then it decreases at the higher frequencies. Using the $f_{\max }$ value, the carrier lifetime $\tau$ was calculated as follows:

$\tau=\frac{1}{2 \pi f_{\max }}$.

The results of electrical characterization of CdTe:Ge:Yb are shown in Table 1. They demonstrate that the semiinsulating CdTe:Ge:Yb with the resistivity as high as $1 \times 10^{9} \Omega \mathrm{cm}$ and the carrier lifetime up to $8 \mu$ s can be obtained for the $\mathrm{Yb}$ concentration in the range of about $1-2 \times 10^{17} \mathrm{~cm}^{-3}$.

Fig. 1 shows the PL spectra of three different CdTe samples doped with $\mathrm{Ge}\left(5 \times 10^{17} \mathrm{at} / \mathrm{cm}^{3}\right)$, doped with $\mathrm{Yb}$ $\left(1 \times 10^{18} \mathrm{at} / \mathrm{cm}^{3}\right)$ and co-doped with Ge:Yb $\left(1 \times 10^{17}\right.$ : $\left.1 \times 10^{18} \mathrm{at} / \mathrm{cm}^{3}\right)$. It is seen that the $\mathrm{Yb}$-doping results in the strong increase of PL intensity. The three spectra exhibit a strong emission of neutral donor-bound excitons in the band-edge region, in which intensity increases with an increase of $\mathrm{Yb}$ concentration. This could imply that the $\mathrm{Yb}$ dopant produces a shift of the Fermi level towards the conduction band, explaining the observed high resistivity of CdTe:Ge:Yb.

Table 1

The electrical resistivity and carrier life-time of CdTe:Ge:Yb

\begin{tabular}{lll}
\hline Yb concentration $\left(\mathrm{at} / \mathrm{cm}^{3}\right)$ & Resistivity $(\Omega \mathrm{cm})$ & Lifetime $(\mu \mathrm{s})$ \\
\hline $1 \times 10^{17}$ & $3 \times 10^{9}$ & 5 \\
$1.5 \times 10^{17}$ & $6 \times 10^{9}$ & 8 \\
$2 \times 10^{17}$ & $2 \times 10^{9}$ & 6 \\
$4 \times 10^{17}$ & $1 \times 10^{7}$ & 6 \\
$5 \times 10^{17}$ & $1 \times 10^{6}$ & 4 \\
$6 \times 10^{17}$ & $2 \times 10^{6}$ & 3 \\
$8 \times 10^{17}$ & $2 \times 10^{6}$ & 2 \\
\hline
\end{tabular}

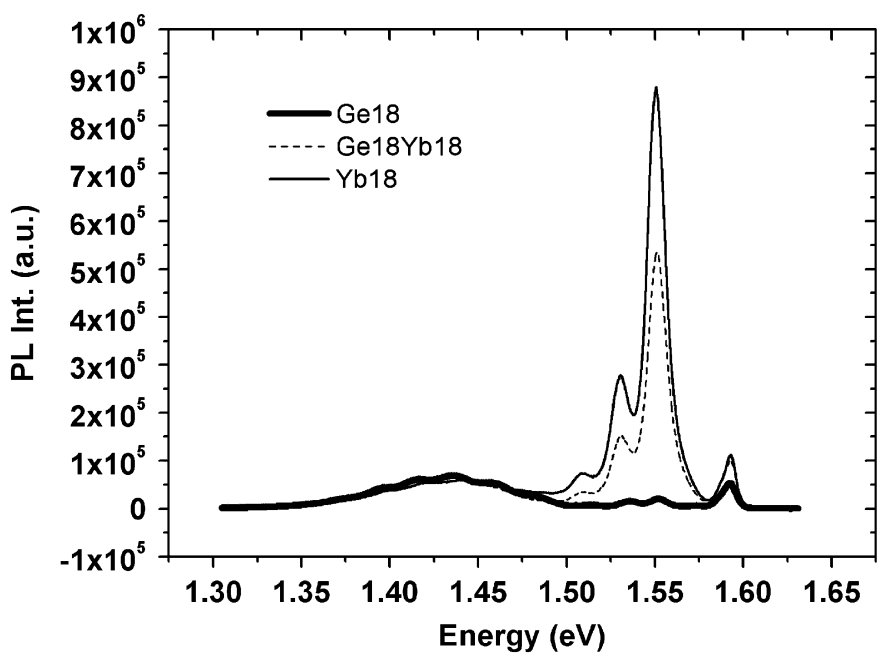

Fig. 1. Photoluminescence spectra of CdTe samples doped with Ge (full line), $\mathrm{Yb}$ (dot line) and co-doped with $\mathrm{Ge}: \mathrm{Yb}$ (dash line) $(T=10 \mathrm{~K})$. 
The other important feature of PL spectra is a strong increase of the PL band centered at $1.55 \mathrm{eV}$, which does not seem to shift in energy with the change of the $\mathrm{Yb}$ concentration. Taking into account that this emission is related with a donor-acceptor pair (DAP) transition between hydrogen-like donor levels and acceptor levels related to defect complex with the structure $\left(V_{\mathrm{Cd}}-D_{\mathrm{Cd}}\right)$ or $\left(V_{\mathrm{Cd}}-2 D_{\mathrm{Cd}}\right)[1,13,14]$, the high intensity of the $1.55 \mathrm{eV}$ band in CdTe:Ge: $\mathrm{Yb}$ and $\mathrm{CdTe}: \mathrm{Yb}$ crystals suggests an increase of donor defects that form complexes with $V_{\mathrm{Cd}}$, confirming that the incorporation of $\mathrm{Yb}$ introduces donor levels in CdTe.

The typical CL image of a high-resistivity CdTe:Ge:Yb bulk crystal is presented in Fig. 2. It exhibits the grained crystal structure in which the grain boundaries appear as dark lines surrounded sometimes by the bright bands at both sides. This contrast, which has been reported in the CL studies of CdTe:Ge as well as other semiconductors and insulator materials $[9,10]$, is due to the decoration of boundaries by impurities and/or point defects. The interior area of grains is very homogeneous without any microscale structural defects like voids, precipitates, impurity clusters, etc. Nevertheless, some of these defects are observed as non-radiative dark spots within the grain boundaries, proving their role as defect segregation centers. This segregation seems to be also related with the purification effect caused by the interaction of $\mathrm{Yb}$ dopant with the group I residual impurities.

The increase of $\mathrm{Yb}$ concentration above the value of about $5 \times 10^{18} \mathrm{~cm}^{-3}$ results in a loss of homogeneity of $\mathrm{CdTe}: \mathrm{Ge}: \mathrm{Yb}$ crystals, with the formation of a higher number of grains and small subgrains in which the bright

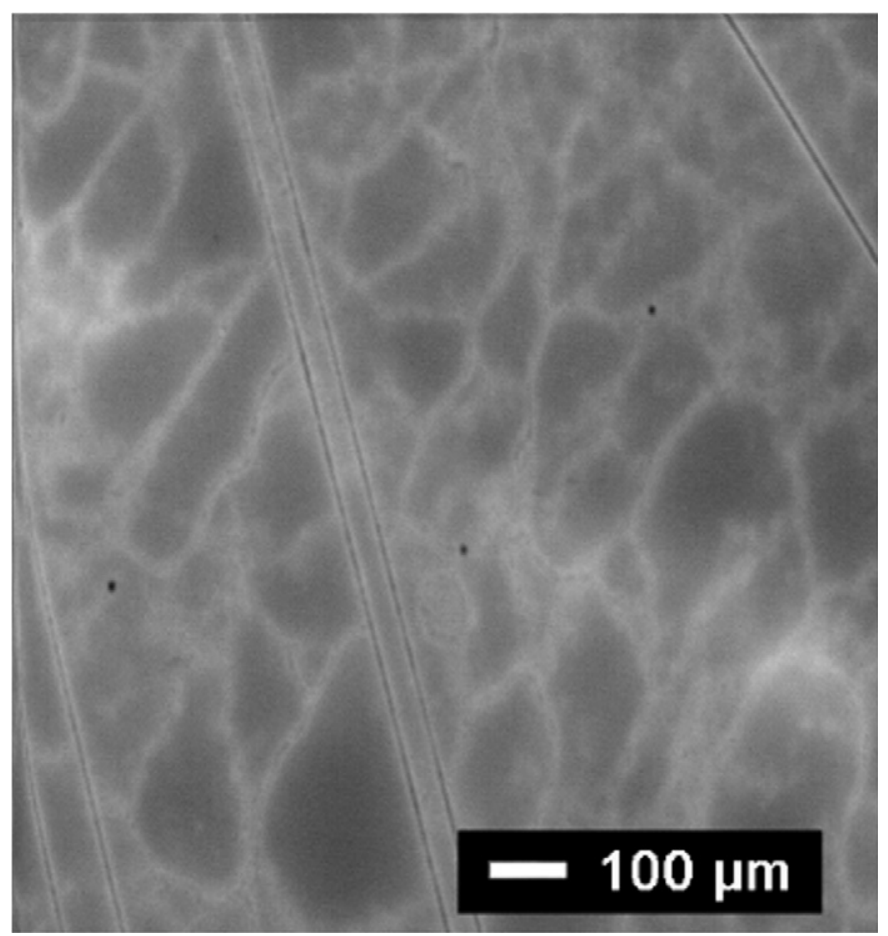

Fig. 2. The CL image of a high-resistivity CdTe:Ge:Yb bulk crystal. bands extend for tens of microns at both sides. It is also worth mentioning that CL images of the CdTe:Ge:Yb VPE layers had very low uniform contrast that did not allow to make any conclusions about the spatial distribution of radiative structural defects.

Fig. 3 presents the CL spectrum of a CdTe:Ge:Yb bulk crystal, that shows several deep level bands. The Gaussian deconvolution of the most intense band centered at $1.1 \mathrm{eV}$ shows that it has several components and it was previously suggested to be connected with tellurium vacancies $[15,16]$.

The CL spectrum of the CdTe:Ge:Yb VPE layer, which is shown in Fig. 4, has a much more complex structure. The influence of $\mathrm{Yb}$ is also apparent in the $1.1 \mathrm{eV}$ band that is more narrow and intense than in the bulk samples and shows a slight shift to higher energies. The Gaussian

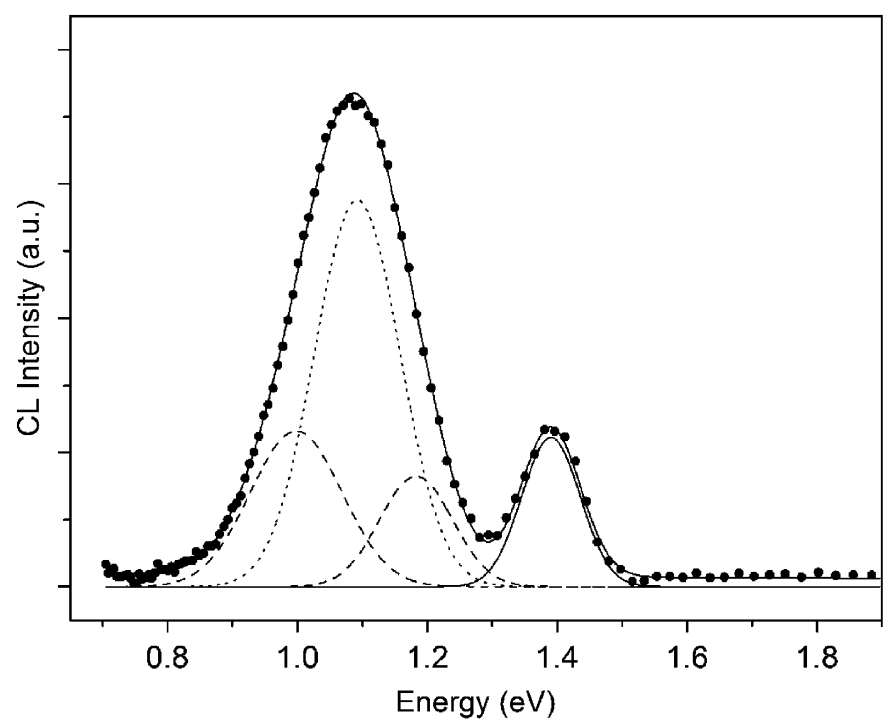

Fig. 3. The CL spectrum of a CdTe:Ge:Yb bulk crystal with the Gaussian deconvolution of the band centered at $1.1 \mathrm{eV}$.

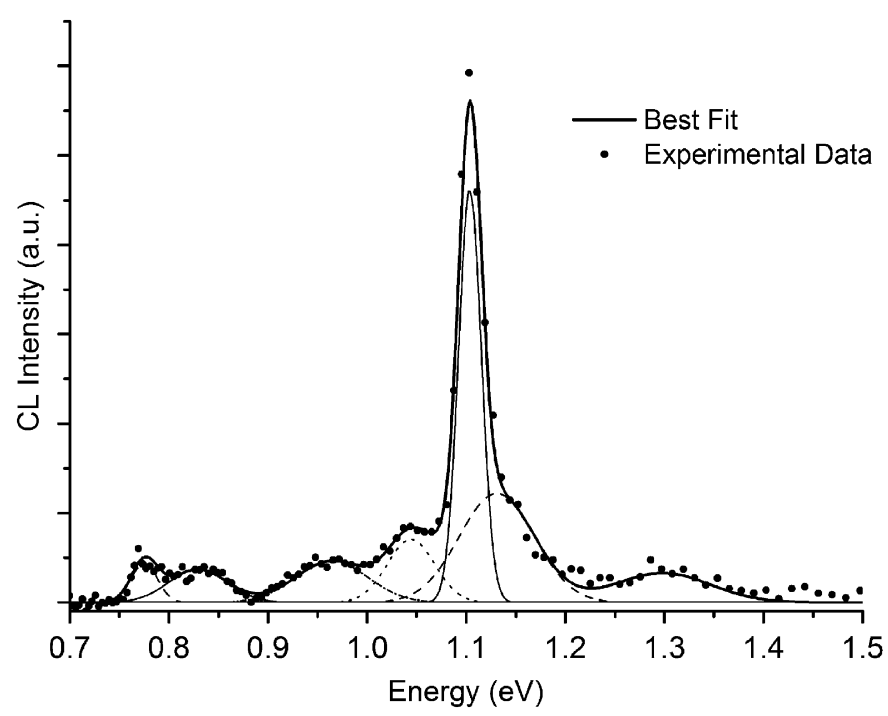

Fig. 4. The CL spectrum of a CdTe:Ge:Yb VPE layer with the Gaussian deconvolution of the band centered at $1.1 \mathrm{eV}$. 
deconvolution of this band shows several components that have not been identified yet.

\section{Conclusions}

The CdTe:Ge:Yb crystals and epitaxial layers were grown by the Bridgman method and VPE, respectively, and their structural, electrical, PL and CL properties were reported. Experimental findings testify that high-resistivity crystals and layers of reasonably good structural quality can be grown with the $\mathrm{Yb}$ concentration below the value of $5 \times 10^{18} \mathrm{~cm}^{-3}$. Further work is in progress to estimate the ability of CdTe:Ge:Yb for X- and gamma-ray detectors.

\section{Acknowledgments}

This work has been partly supported by the projects CAM SENSORCDT S-0505/MAT/0209 and EU FP6 PHOLOGIC 017158.

\section{References}

[1] N.V. Sochinskii, V.N. Babentsov, E. Diéguez, Cadmium telluride and related compounds, in: D.R. Vij, N. Singh (Eds.), Physics and Chemistry of II-VI Luminescence Semiconductors, NOVA Science Publishers, Inc., New York, USA, 1996, pp. 248-276 (Chapter VI).
[2] M. Schieber, et al., J. Crystal Growth 231 (2001) 235.

[3] E. Saucedo, L. Fornaro, N.V. Sochinskii, A. Cuña, V. Corregidor, D. Granados, E. Diéguez, IEEE Trans. Nucl. Sci. 51 (2004) 3105.

[4] S. Neretina, N.V. Sochinskii, P. Mascher, E. Saucedo, Mater. Res. Soc. Symp. Proc. 864 (2005) E4.18.1.

[5] E. Saucedo, C.M. Herrero, L. Fornaro, N.V. Sochinskii, E. Diéguez, J. Crystal Growth 275 (2005) 471.

[6] E. Saucedo, O. Martínez, C.M. Ruiz, O. Vigil-Galán, I. Benito, L. Fornaro, N.V. Sochinskii, E. Diéguez, J. Crystal Growth 291 (2006) 416.

[7] M. Fiederle, V. Babentsov, J. Franc, A. Fauler, K.W. Benz, R.B. James, E. Cross, J. Crystal Growth 243 (2002) 77.

[8] J. Franc, P. Horodyský, R. Grill, J. Kubát, E. Saucedo, N.V. Sochinskii, J. Crystal Growth 286 (2006) 384.

[9] U. Pal, P. Fernandez, J. Piqueras, N.V. Sochinskii, E. Diéguez, J. Appl. Phys. 78 (1995) 1992.

[10] G. Panin, J. Piqueras, N.V. Sochinskii, E. Dieguez, Appl. Phys. Lett. 70 (1997) 877.

[11] N.V. Sochinskii, M. Lozano, G. Pellegrini, M. Ullan, Nucl. Instru. and Methods A 568 (2006) 451.

[12] D. Wagner, P. Irsigler, D.J. Dunstan, J. Phys. C 17 (1984) 6793.

[13] J. Aguilar-Hernández, M. Cárdenas-García, G. Contreras-Puente, J. Vidal-Larramendi, Mater. Sci. Eng. B 102 (2003) 203.

[14] S.H. Song, J. Wang, Y. Ishikawa, S. Seto, M. Isshiki, J. Crystal Growth 237-239 (2002) 1726.

[15] Z. Sobiesierski, I.M. Dharmadasa, R.H. Williams, Appl. Phys. Lett. 53 (1988) 2623.

[16] C.B. Davis, D.D. Allred, A. Reyes-Mena, J. González-Hernández, O. González, B.C. Hess, W.P. Allred, Phys. Rev. B 47 (1993) 13363. 